\title{
Glycogen Hepatopathy: A Rare and Underrecognized Cause of Recurrent Transaminitis in Patients with Uncontrolled Type 2 Diabetes Mellitus
}

\author{
Kishore Kumar Shehriyar Mehershahi Chukwunonso Chime \\ Hassan Tariq Suresh Kumar Nayudu Sridhar Chilimuri
}

Division of Gastroenterology, Department of Medicine, Bronx Lebanon Hospital Center, Bronx, NY, USA

\section{Keywords}

Glycogen hepatopathy · Recurrent transaminitis · Type 2 diabetes mellitus

\begin{abstract}
Glycogen hepatopathy $(\mathrm{GH})$, characterized by reversible transaminitis and hepatomegaly, results from excessive accumulation of glycogen in hepatocytes. GH has been well described in the literature as a rare cause of transaminitis in children and young patients with uncontrolled type 1 diabetes mellitus and has rarely been reported in type 2 diabetic patients. Hyperglycemia and hyperinsulinemia are believed to be a metabolic substrate for hepatic glycogen accumulation and in order to cause glycogen hepatotoxicity. We present the case of a 54-year-old woman with poorly controlled insulin-dependent type 2 diabetes who was hospitalized twice within 1 month with diabetic ketoacidosis/hyperosmolar hyperglycemic state and reversible transaminitis. Interestingly, she had normal liver function tests performed at the time of admission and transaminitis was noted 1 day later, after she was treated with high doses of intravenous insulin therapy. Subsequently, liver enzymes recovered to normal levels with optimization of glucose control.




\section{Case Reports in Gastroenterology}

Case Rep Gastroenterol 2018;12:466-472

DOI: $10.1159 / 000492205$

(c) 2018 The Author(s). Published by S. Karger AG, Base www.karger.com/crg

Kumar et al.: Glycogen Hepatopathy: A Rare and Underrecognized Cause of Recurrent

Transaminitis in Patients with Uncontrolled Type 2 Diabetes Mellitus

\section{Introduction}

Glycogen hepatopathy (GH), characterized by reversible transaminitis and hepatomegaly, results from excessive accumulation of glycogen in hepatocytes. Mauriac [1] first defined GH in a child with brittle diabetes, as a component of Mauriac syndrome, characterized by delayed development, hepatomegaly, cushingoid appearance, and delayed puberty. GH has been well described as a rare cause of transaminitis in young patients with uncontrolled type 1 diabetes mellitus and has rarely been reported in type 2 diabetic patients. Hyperglycemia and hyperinsulinemia are believed to be a metabolic substrate for hepatic glycogen accumulation and in order to cause glycogen hepatotoxicity. Nonalcoholic fatty liver disease (NAFLD) is the most common cause of chronic liver disease for both the general and the diabetic populations currently due to its association with obesity and metabolic syndrome. Clinician awareness of GH should prevent diagnostic delay and will provide better insight into the prevalence of GH. In addition, the distinction between GH and NAFLD is important, as prognosis of both diseases may be different $[1,2]$.

\section{Case Presentation}

A 54-year-old Hispanic woman presented to our emergency room with complaints of abdominal pain and nausea for 2 days. The patient denied fever, vomiting, diarrhea, yellow discoloration, sick contact, recent traveling, Tylenol ingestion, and she denied a history of herbal medication use. Her medical history was significant for diabetes mellitus type 2, asthma, depression, chronic back pain, and laparoscopic cholecystectomy. Her medications included insulin aspart 30 units 3 times daily with meals, insulin Lantus 35 units at bedtime, aspirin 81 mg once daily, albuterol inhaler as needed, fluticasone/salmeterol twice daily, and clonazepam daily. She had no family history of diabetes mellitus or liver disease. She reported cigarette smoking and denied alcohol and drug use. On presentation, her temperature was $97.9^{\circ} \mathrm{F}$, blood pressure $143 / 92 \mathrm{~mm} \mathrm{Hg}$, pulse 97 beats per minute, respiratory rate 16 breaths per minute, oxygen saturation $98 \%$ on room air, and her BMI was 24 . Physical exam was unremarkable except for mild hepatomegaly. Initial laboratory investigations were consistent with a hyperosmolar hyperglycemic state with normal liver enzyme levels, and she was started on intravenous insulin therapy along with aggressive fluid resuscitation. On the second day of hospitalization, she was noted to have elevated liver enzyme levels with aspartate transaminase/alanine aminotransferase (AST/ALT) of 424/145 U/L and alkaline phosphatase (ALP) of $187 \mathrm{U} / \mathrm{L}$ (Table 1). However, liver enzymes improved on day 4, with optimization of her blood sugar levels and substitution of intravenous insulin with subcutaneous insulin.

Three weeks prior to this admission, she was admitted to the hospital with diabetic ketoacidosis and normal liver function tests at the time of admission. One day after starting intravenous insulin, she developed transaminitis with AST of $665 \mathrm{U} / \mathrm{L}$ and ALT of $231 \mathrm{U} / \mathrm{L}$ that also resolved to normal values with optimization of blood sugar levels and upon transition from intravenous insulin to subcutaneous insulin. Hepatitis workup, including viral hepatitis A, B, C, iron profile, anti-neutrophil antibody, anti-mitochondrial antibody, celiac panel, serum ceruloplasmin, anti-smooth muscle antibody, and anti-liver kidney muscle antibody, was 


\section{Case Reports in Gastroenterology}

Case Rep Gastroenterol 2018;12:466-472

DOI: $10.1159 / 000492205$

(c) 2018 The Author(s). Published by S. Karger AG, Basel www.karger.com/crg

Kumar et al.: Glycogen Hepatopathy: A Rare and Underrecognized Cause of Recurrent

Transaminitis in Patients with Uncontrolled Type 2 Diabetes Mellitus

unremarkable (Table 2). Ultrasound of the abdomen showed hepatomegaly with normal liver contour and echo pattern. Subsequent magnetic resonance pancreatography revealed no biliary tree pathology.

Ischemic hepatopathy can cause acute appearance and rapid amelioration of liver enzymes; however, there was no episode of hypotension during both hospitalizations. Moreover, temporal relationship with insulin therapy in the setting of hyperglycemia was more suggestive of a diagnosis of GH. Similarly, NAFLD was considered an alternative cause of transaminitis due to her history of diabetes, dyslipidemia, and obesity. However, NAFLD rarely causes aminotransferase elevation similar to the one observed in this patient. The acute increase in transaminases immediately after starting insulin therapy and rapid normalization of liver enzymes is uncommon in NAFLD. Nonetheless, the presence of some degree of underlying fatty infiltration could not be ignored in this case [5]. Other common and possible etiologies which could have contributed to transaminitis include alcoholic liver disease, drug-induced liver injury, and severe sepsis and were excluded due to the absence of alcohol use, known hepatoxic drug, and underlying sepsis, respectively. A liver biopsy is a definitive way to diagnose GH, but the patient did not agree to the procedure.

\section{Discussion}

Transaminitis is a very common laboratory abnormality in patients with diabetes mellitus, obesity, and metabolic syndrome. The prevalence of elevated ALT level is $9.5 \%$ among type 1 and $12.1 \%$ among type 2 diabetics. These percentages are higher than those expected in the general population $(2.7 \%)$. NAFLD is currently the most common cause of chronic liver disease for both the general and the diabetic populations due to its association with obesity and metabolic syndrome. Therefore, NAFLD was observed more frequently in type 2 diabetes cases compared to type 1 diabetes. However, it should be noted that NAFLD is a diagnosis of exclusion [1].

$\mathrm{GH}$, a disease that develops due to excessive hepatic glycogen accumulation, is characterized by hepatomegaly and elevated liver enzyme levels. GH was first described in 1930, as a component of Mauriac syndrome, which includes type 1 diabetes, delayed development, hepatomegaly, cushingoid appearance, and delayed puberty [1]. However, it can also be observed in type 1 diabetic individuals without the other components of Mauriac syndrome. Biochemically, the glycogen accumulation takes place when hyperglycemia activates glycogen synthase enzyme by inhibiting glycogenolysis via glycogen phosphorylation inactivation. Inside the cell, glucose is converted into glucose-6-phosphate and ultimately to glycogen by enzyme glycogen synthase. The enzyme glycogen synthase is converted from an inactive (phosphorylated) form into the active (dephosphorylated) form by a phosphatase. The phosphatase concentration is maintained by insulin and relies on the presence of glucose [3-5]. Hepatic glycogen levels are regulated by balance between glycogenesis and glycogenolysis. The entry of glucose into hepatocytes is due to passive diffusion independent of insulin levels. Therefore, frequent hyperglycemic episodes followed by excessive insulin use in order to optimize the glucose level are believed to be the primary pathogenic mechanism of hepatomegaly and transaminitis in poorly controlled diabetic patients. Accumulation of glycogen in the liver occurs when high insulin levels activate glycogen synthase, which further accelerates the 


\section{Case Reports in Gastroenterology}

Case Rep Gastroenterol 2018;12:466-472

DOI: $10.1159 / 000492205$

(c) 2018 The Author(s). Published by S. Karger AG, Base www.karger.com/crg

Kumar et al.: Glycogen Hepatopathy: A Rare and Underrecognized Cause of Recurrent

Transaminitis in Patients with Uncontrolled Type 2 Diabetes Mellitus

biochemical process of glycogen deposition. Liver biopsy is mandatory for the diagnosis of GH and NAFLD. Persistent and relatively mild disturbance in liver function favors NAFLD; transaminase flares are more compatible with GH.

The hallmark of GH is its reversibility with improved glycemic control, unlike hepatic steatosis; glycogen overload is not known to progress to fibrosis, distinct from fatty liver disease [4]. In addition, ultrasound findings are not helpful in distinguishing NAFLD from GH because both fatty liver and glycogen overload could show a similar ultrasound finding as mild hyperechoic hepatic parenchyma. Consequently, the condition is often misdiagnosed, and it results in wasting medical resources. A final distinction can be made with a liver biopsy. In general, GH is characterized by several histological features which include (1) marked glycogen accumulation. After conventional tissue preparation (fixation by formaldehyde solution and staining with hematoxylin and eosin), the glycogen is usually removed from the hepatocytes. Thus, the hepatocytes are diffusely swollen with a pale cytoplasm and accentuation of the cell membranes, frequently with displacement of the nuclei to the cell periphery, the sinusoids are compressed by swollen hepatocytes, and glycogenated nuclei and giant mitochondria are present; glycogen accumulation within hepatocytes is demonstrated by periodic acid-Schiff staining which disappeared after digestion with diastase; (2) no or a minimal change in fat content; (3) the absence of or minimal inflammation; (4) the absence or minimal presence of spotty lobular necrosis; and (5) intact liver architecture without or with minimal fibrosis [5]. Although GH is definitively diagnosed histologically, a gradient dual-echo magnetic resonance imaging sequence combined with computed tomography of the liver is a powerful methodology for distinguishing GH from NAFLD [5]. The correct diagnosis of this disease is important given its potential resolution after improved glycemic control. It is essential to differentiate GH from other liver disorders, including NAFLD, to avoid unnecessary workup, prevent diagnostic delay, and provide high-value care.

\section{Conclusion}

Based on concomitant elevation of transaminases with hyperglycemia and temporal association with insulin therapy, it is reasonable to conclude that this intermittent transaminase elevation is secondary to GH. Nonetheless, GH cannot be confirmed clinically or by ultrasound alone and it always requires liver biopsy for a definitive diagnosis. The reversibility of transaminase elevations in this patient indicates a more benign course of $\mathrm{GH}$. Clinician awareness of GH should prevent diagnostic delay and will provide better insight into the prevalence of GH. In addition, the distinction between GH and NAFLD is important, as the prognosis of both diseases may be different. GH has been known to have a benign nature, whereas NAFLD could progress into advanced liver disease such as cirrhosis or hepatocellular carcinoma [2]. This case highlights this underdiagnosed entity in type 2 diabetics with acute relapsing hepatitis, hence prompting the clinician to consider this condition in the differential diagnosis as one of the possible etiologies of reversible liver injury. 


\section{Case Reports in Gastroenterology}

Case Rep Gastroenterol 2018:12:466-472

DOI: $10.1159 / 00049220$

(c) 2018 The Author(s). Published by S. Karger AG, Basel www.karger.com/crg

Kumar et al.: Glycogen Hepatopathy: A Rare and Underrecognized Cause of Recurrent

Transaminitis in Patients with Uncontrolled Type 2 Diabetes Mellitus

\section{Statement of Ethics}

Ethics approval: not obtained.

Consent for publication: consent obtained.

Patient's confidentiality is maintained.

\section{Disclosure Statement}

The authors declare that they have no competing interests. This case has never been published before and the authors have no conflict of interest.

\section{Funding Sources}

This paper was not funded.

\section{Author Contributions}

All authors have contributed to writing and reviewing the manuscript.

\section{References}

1 Mauriac P. Gros ventre, hepatomegalie, troubles de las croissance chez les enfants diabetiques traits depuis plusieurs annes par l'insuline. Gax Hebd Med Bordeaux. 1930;26:402-10.

2 Atmaca M, Ucler R, Kartal M, Seven I, Alay M, Bayram I, et al. Glycogenic Hepatopathy in Type 1 Diabetes Mellitus. Case Reports Hepatol. 2015;2015:236143.

3 Cha JH, Ra SH, Park YM, Ji YK, Lee JH, Park SY, et al. Three cases of glycogenic hepatopathy mimicking acute and relapsing hepatitis in type I diabetes mellitus. Clin Mol Hepatol. 2013 Dec;19(4):421-5.

4 Messeri S, Messerini L, Vizzutti F, Laffi G, Marra F. Glycogen hepatopathy associated with type 1 diabetes mellitus as a cause of recurrent liver damage. Ann Hepatol. 2012 Jul-Aug;11(4):554-8.

5 Julián MT, Alonso N, Ojanguren I, Pizarro E, Ballestar E, Puig-Domingo M. Hepatic glycogenosis: An underdiagnosed complication of diabetes mellitus? World J Diabetes. 2015 Mar 15;6(2):321-5. 


\section{Case Reports in Gastroenterology}

\begin{tabular}{l|l}
\hline Case Rep Gastroenterol 2018;12:466-472 \\
\hline DOI: 10.1159/000492205 & $\begin{array}{l}\text { ○ 2018 The Author(s). Published by S. Karger AG, Basel } \\
\text { www.karger.com/crg }\end{array}$ \\
\hline
\end{tabular}

Kumar et al.: Glycogen Hepatopathy: A Rare and Underrecognized Cause of Recurrent Transaminitis in Patients with Uncontrolled Type 2 Diabetes Mellitus

Table 1. Biochemical testing at second admission

\begin{tabular}{lllll}
\hline & Day 1 & Day 2 & Day 4 & After discharge \\
\hline Serum glucose, mg/dL & 808 & 104 & 98 & 275 \\
Anion gap, mmol/L & 15 & 12 & 14 & 17 \\
Lactic acid, mmol/L & 1.1 & 0.9 & & \\
Bicarbonate, mEq/L & 23 & 24 & 24 & 26 \\
Sodium, mEq/L & 122 & 138 & 142 & 139 \\
Potassium, mEq/L & 4.1 & 3.4 & 3.6 & 3.4 \\
Serum ketone (qualitative) & +++ & & & \\
Alanine aminotransferase, U/L & 15 & 145 & 69 & 14 \\
Aspartate transaminase, U/L & 10 & 424 & 33 & 13 \\
Alkaline phosphatase, U/L & 186 & 187 & 147 & 186 \\
Total bilirubin, mg/dL & 0.3 & 0.2 & 0.2 & 0.4 \\
Ethanol, mg/dL & $<10$ & & & \\
\hline
\end{tabular}


Kumar et al.: Glycogen Hepatopathy: A Rare and Underrecognized Cause of Recurrent Transaminitis in Patients with Uncontrolled Type 2 Diabetes Mellitus

Table 2. Laboratory evaluation showing workup done during the first admission to evaluate the cause of abnormal liver function tests

\begin{tabular}{llrr}
\hline & Day 1 & Day 2 & Discharge \\
\hline Serum glucose, mg/dL & 779 & 157 & 140 \\
Anion gap, mmol/L & 23 & 9 & 10 \\
Lactic acid, mmol/L & 1.4 & 0.9 & 1.1 \\
Serum bicarbonate, mEq/L & 18 & 25 & 23 \\
Sodium, mEq/L & 125 & 135 & 139 \\
Potassium, mEq/L & 4.7 & 3.0 & 4.0 \\
HbAtc, \% & 22.6 & & 15 \\
Alanine aminotransferase, U/L & 24 & 231 & 10 \\
Aspartate transaminase, U/L & 16 & 665 & \\
Alkaline phosphatase, U/L & 187 & 162 & \\
Total bilirubin, mg/dL & 0.3 & 0.3 & \\
Hepatitis A IgG & Positive & & \\
Hepatitis A IgM & Negative & & \\
Hepatitis B surface antigen & Negative & & \\
Hepatitis B surface antibody & Positive & & \\
Ethanol, mg/dL & $<10$ & & \\
Anti-hepatitis C antibody & Negative & & \\
Ferritin, ng/mL & & 194 \\
Anti-neutrophil antibody & Negative & & \\
Anti-mitochondrial antibody & Negative & & \\
Serum acetone (qualitative) & Positive & & \\
Serum ceruloplasmin, mg/dL & 26 & \\
HIV & Negative & & \\
Anti-smooth muscle antibody & Negative & & \\
Liver kidney microsomal assay $\leq 20$ & Negative & & \\
\hline
\end{tabular}

